\title{
Simulation of binary mixtures with the lattice Boltzman method
}

\author{
S. Arcidiacono* and J. Mantzaras ${ }^{\dagger}$ \\ Paul Scherrer Institute, Combustion Research, CH-5232 Villigen PSI, Switzerland \\ S. Ansumali \\ Nanyang Technological University, School of Chemical and Biomedical Engineering, Singapore 639798
}

I. V. Karlin, ${ }^{\S}$ C. Frouzakis," and K. B. Boulouchos ${ }^{\mathbb{T}}$

ETH-Zürich, Institute of Energy Technology, CH-8092 Zürich, Switzerland

(Received 12 May 2006; revised manuscript received 28 September 2006; published 22 November 2006)

\begin{abstract}
A lattice Boltzman model for the simulation of binary mixtures is presented. Contrary to previous models, the present formulation is able to simulate mixtures with different Schmidt numbers and arbitrary molecular mass ratio of the components. In the hydrodynamic limit, the Navier-Stokes and the Stefan-Maxwell binary diffusion equations are recovered. The model is used for the simulation of binary diffusion and mixing layers. The results are found to be in good agreement with a derived similarity solution and with the predictions of a transient spectral element code.

DOI: 10.1103/PhysRevE.74.056707

PACS number(s): 47.11.- j, 05.20.Dd
\end{abstract}

\section{INTRODUCTION}

Mixing is an important process in many practical applications such as chemical reactions, pollutant dispersion, diffusion in porous media, etc. Traditionally, mass and momentum transfer are modeled using the continuum assumption. However, as the Knudsen number Kn increases and the characteristic hydrodynamic length becomes comparable to the mean free path of the gases, the continuum assumption breaks down and the use of kinetic theory is required. Recent successes of kinetic algorithms like the lattice Boltzman method [1-8] have shown that the use of kinetic theory may be beneficial in the continuum domain as well. It seems natural, therefore, to extend the LB approach to mixtures. Indeed, recently some LB models have been proposed for mixtures (for a recent review, see Ref. [9]). Such attempts were based either on a passive scalar model, or on direct discretization of BGK-type models derived from the continuous kinetic theory. Most of those models satisfy neither the $H$-theorem nor the indifferentiability principle, i.e. they do not reduce to the single-component BGK fluid when the species become mechanically equivalent (see, e.g. Refs. [9,10]). Furthermore, the existing lattice Boltzman models for mixtures face severe numerical instabilities at large molecular mass ratios [11].

An extended formulation of the LB model for binary mixtures initially introduced in Ref. [12] is presented in this paper. The model ensures: thermodynamic consistency (the $H$-theorem is satisfied) $[13,14]$, the indifferentiability principle, the recovering of the Navier-Stokes and the Stefan-

\footnotetext{
*Email address: salvatore.arcidiacono@psi.ch

†Email address: ioannis.mantzaras@psi.ch

‡Email address: ansumali@gmail.com

${ }^{\S}$ Corresponding author. Email address: ikarlin@lav.mavt.ethz.ch

"Email address: frouzakis@lav.mavt.ethz.ch

IIEmail address: boulouchos@lav.mavt.ethz.ch
}

Maxwell diffusion equations, a straightforward generalization to a multiple species formulation, and the capability to simulate large molecular mass ratios. As an example of model flexibility, results are presented for the interdiffusion of two species $A$ and $B$ with a molecular mass ratio $m_{B} / m_{A}$ $=500$, followed by a demanding dynamic simulation (mixing layer) that is further compared with a similarity solution, derived for the purposes of this work, and with the predictions of a spectral element code that solves for the NavierStokes (NS) and species conservation equations.

The model is described in detail in Secs. II-IV. Section V provides the numerical implementation of the model. In Sec. VI two different applications are considered and the results from the LB code are compared either with analytical solutions or with predictions of a continuum code. Conclusions are reported in Sec. VII.

\section{MODEL}

The discrete-velocity kinetic equation for each component $j=A, B$ of a binary mixture can be written as

$$
\partial_{t} f_{j i}+c_{j i \alpha} \partial_{\alpha} f_{j i}=\Omega_{j i}
$$

where $i=0, \ldots, N$, with $N$ number of the discrete lattice velocities $c_{j i \alpha}, \alpha=\{x, y, z\}$ and $\Omega_{j i}$ is the collision term. In Eq. (1) and subsequently, summation convention is applied to the index of the spatial direction.

The basic concept of the proposed kinetic model is a representation on the fast-slow decomposition of motions near quasi-equilibrium states, as proposed in Ref. [14]. The relaxation to equilibrium is modeled as a two-step process, a "fast" relaxation from the initial state $f$ to the quasiequilibrium $f^{*}$, and a "slow" motion from the quasiequilibrium state $f^{*}$ towards the equilibrium $f^{\text {eq }}$. This decomposition is thermodynamically consistent since it ensures a non-negative entropy production [14] (a proof for the present model is provided in the Appendix). Both motions can be approximated by a BGK term [15] with the condition $\tau_{2}$ 
$\geqslant \tau_{1}$, where $\tau_{1}$ and $\tau_{2}$ are the relaxation times from $f$ to $f^{*}$ and from $f^{*}$ to $f^{\text {eq }}$, respectively. Under these assumptions, the collision integral $\Omega_{j i}$ can be expressed as

$$
\Omega_{j i}=\frac{1}{\tau_{1}}\left[f_{j i}^{*}-f_{j i}\right]+\frac{1}{\tau_{2}}\left[f_{j i}^{\mathrm{eq}}-f_{j i}^{*}\right] .
$$

By definition, the moments of each component $j$ are

$$
\rho_{j}=\sum_{i}^{N} f_{j i}, \quad J_{j \alpha}=\sum_{i}^{N} f_{j i} c_{j i \alpha}
$$

$$
P_{j \alpha \beta}=\sum_{i}^{N} f_{j i} c_{j i \alpha} c_{j i \beta}, \quad Q_{j \alpha \beta \gamma}=\sum_{i}^{N} f_{j i} c_{j i \alpha} c_{j i \beta} c_{j i \gamma},
$$

where $\rho_{j}, J_{j \alpha}, P_{j \alpha \beta}$, and $Q_{j \alpha \beta \gamma}$ are the density, the momentum, the pressure tensor, and the third-order moment of component $j$, respectively.

As usual in the entropic LB approach, the $H$-function is defined according to:

$$
H=\sum_{j}^{A, B} \sum_{i}^{N} f_{j i} \ln \frac{f_{j i}}{W_{i}}
$$

where $W_{i}$ are weights associated to the corresponding discrete lattice velocities [5]. In the present study the D2Q9 velocity model is implemented, and the discrete lattice velocities vectors are

$$
c_{j i}=\left\{\begin{array}{ll}
c_{j}\{0,0\} & i=0, \\
c_{j} \sqrt{2}\left\{\left(\cos \left(\frac{\pi(i-1)}{2}\right), \sin \left(\frac{\pi(i-1)}{2}\right)\right\}\right. & i=1 \text { to } 4 .
\end{array} .\right.
$$

The magnitudes of the discrete lattice velocities, $c_{j}$, are related to the speed of sound $c_{s j}=\sqrt{k_{B} T_{0} / m_{j}}$ of each component as $c_{j}=\sqrt{3} c_{s j}$, where $m_{j}$ is the molecular weight of component $j, k_{B}$ is the Boltzman constant, and $T_{0}$ is a reference temperature. The corresponding weight vector is

$$
W_{i}= \begin{cases}\frac{4}{9} & \text { if } i=0 \\ \frac{1}{9} & \text { if } i=1 \text { to } 4 \\ \frac{1}{36} & \text { if } i=5 \text { to } 8 .\end{cases}
$$

The equilibrium distribution function is evaluated by minimizing Eq. (4) under the constraint of conservation of the density of each species $\left(\rho_{j}\right)$, and of the total mixture momentum $\mathbf{J}=\mathbf{J}_{A}+\mathbf{J}_{B}$ (the energy conservation is not relevant in the present isothermal model):

$$
\rho_{j}=\sum_{i}^{N} f_{j i}, \quad J_{\alpha}=\sum_{j}^{A, B} \sum_{i}^{N} f_{j i} c_{j i \alpha} .
$$

The result of the minimization problem can be directly extracted from the equilibrium of a single-component fluid [5]:

$$
\begin{aligned}
f_{j i}^{\mathrm{eq}}\left(\rho_{j}, \mathbf{U}\right)= & \rho_{j} W_{i} \prod_{\alpha=1}^{d}\left(\frac{2 c_{j}-\sqrt{c_{j}^{2}+3 U_{\alpha}^{2}}}{c_{j}}\right) \\
& \times\left(\frac{2 U_{\alpha}+\sqrt{c_{j}^{2}+3 U_{\alpha}^{2}}}{c_{j}-U_{\alpha}}\right)^{c_{j i \alpha} / c_{j}},
\end{aligned}
$$

where $d$ is the spatial dimensionality of the system, $U_{\alpha}$ $=J_{\alpha} / \rho$ is the velocity of the mixture in the $\alpha$ direction, and $\rho=\rho_{A}+\rho_{B}$.

The quasi-equilibrium distribution function $f_{j}^{*}$ of each component $j$ is obtained by minimizing the $H$-function under the same constraints of the conserved variables and some additional linear constraints (quasi-conserved variables) that are considered to approach the equilibrium slowly, while all other moments are assumed to equilibrate faster. The quasiequilibrium used in the present implementation is obtained by minimizing the $H$-function using as a linear constraint the difference between the momentum $J_{\alpha}$ of the mixture and the momentum $J_{j \alpha}$ of component $j$. The constraints for this case can be written as

$$
\begin{gathered}
\rho_{j}=\sum_{i}^{N} f_{j i}, \\
J_{\alpha}=\sum_{j}^{A, B} \sum_{i}^{N} f_{j i} c_{j i \alpha},
\end{gathered}
$$




$$
J_{\alpha}-J_{j \alpha}=\sum_{j}^{A, B} \sum_{i}^{N} f_{j i} c_{j i \alpha}-\sum_{i}^{N} f_{j i} c_{j i \alpha},
$$

which is equivalent to the set of constraints

$$
\begin{gathered}
\rho_{j}=\sum_{i}^{N} f_{j i}, \\
J_{j \alpha}=\sum_{i}^{N} f_{j i} c_{j i \alpha} .
\end{gathered}
$$

It can be easily shown that the minimization problem is the same as in the one-component case, and that the quasiequilibrium populations are obtained by substituting in Eq. (8) the velocity $\mathbf{U}_{j}=\mathbf{J}_{j} / \rho_{j}$ of each component

$$
f_{j i}^{*}\left(\rho_{j}, \mathbf{U}_{j}\right)=f_{j i}^{\mathrm{eq}}\left(\rho_{j}, \mathbf{U}_{j}\right) .
$$

As shown below, the choice of this quasi-equilibrium leads to a limitation on the Schmidt number $\mathrm{Sc}=\nu / D_{A B}$, where $\nu$ is the kinematic viscosity and $D_{A B}$ is the binary diffusion coefficient: $\mathrm{Sc} \leqslant \mathrm{Sc}^{*}$, where $\mathrm{Sc}^{*}$ is a reference Schmidt number to be defined at the end of Sec. IV. Extension of the model to $\mathrm{Sc}>\mathrm{Sc}^{*}$ will be briefly discussed in the same section. The standard BGK model for a single-component fluid is obtained from Eqs. (2) and (11) when $\tau_{1}=\tau_{2}$ and $m_{A}=m_{B}$ (the indifferentiability principle is recovered).

\section{HYDRODYNAMICS}

In order to relate the momentum $J_{j \alpha}$ of component $j$ in the $\alpha$ direction to the total momentum $J_{\alpha}$ of the mixture, a diffusion flux $V_{\alpha}$ is defined as

$$
V_{\alpha}=m_{A B}\left(\frac{J_{A \alpha}}{\rho_{A}}-\frac{J_{B \alpha}}{\rho_{B}}\right),
$$

with $m_{A B}=\rho_{A} \rho_{B} /\left(\rho_{A}+\rho_{B}\right)$ denoting the reduced mass.

Using the definition of Eq. (12):

$$
\begin{aligned}
& J_{A \alpha}=\frac{\rho_{A}}{\rho} J_{\alpha}+V_{\alpha}, \\
& J_{B \alpha}=\frac{\rho_{B}}{\rho} J_{\alpha}-V_{\alpha} .
\end{aligned}
$$

Multiplying Eqs. (1) by $\left[1, c_{j i \alpha}, c_{j i \alpha} c_{j i \beta}\right]$, summing up and further using Eqs. (3) and (13), the moment transport equations for each component $j$ are recovered in the hydrodynamic limit.

Density:

$$
\partial_{t} \rho_{j}+\partial_{\alpha}\left(\frac{\rho_{j}}{\rho} J_{\alpha} \pm V_{\alpha}\right)=0 .
$$

Momentum:

$$
\partial_{t} J_{j \alpha}+\partial_{\beta} P_{j \alpha \beta}=\mp \frac{1}{\tau_{2}} V_{\alpha} .
$$

Pressure:

$$
\begin{aligned}
\partial_{t} P_{j \alpha \beta}+\partial_{\gamma} Q_{j \alpha \beta \gamma}= & \frac{1}{\tau_{1}}\left[P_{j \alpha \beta}^{*}\left(\rho_{j}, \mathbf{J}_{j}\right)-P_{j \alpha \beta}\right]+\frac{1}{\tau_{2}}\left[P_{j \alpha \beta}^{\mathrm{eq}}\left(\rho_{j}, \mathbf{J}\right)\right. \\
& \left.-P_{j \alpha \beta}^{*}\left(\rho_{j}, \mathbf{J}_{j}\right)\right] .
\end{aligned}
$$

$P_{j \alpha \beta}^{*}\left(\rho_{j}, \mathbf{J}_{j}\right)$ is the partial pressure of component $j$ at quasiequilibrium and $P_{j \alpha \beta}^{\mathrm{eq}}\left(\rho_{j}, \mathbf{J}\right)$ is the same quantity at equilibrium. The upper sign in Eqs. (14) and (15) corresponds to component $A$, while the lower to component $B$.

In this formulation, since there are no chemical reactions, only the density of each component is conserved, while the momentum and the pressure tensor are nonconserved moments. In the following derivation only the equations pertinent to the mixture will be considered. The transport equations for the mixture density, the density difference, and the mixture momentum are obtained by adding or subtracting the corresponding species equations,

$$
\begin{gathered}
\partial_{t} \rho+\partial_{\alpha} J_{\alpha}=0, \\
\partial_{t}\left(\rho_{A}-\rho_{B}\right)+\partial_{\alpha}\left[\left(\rho_{A}-\rho_{B}\right) \frac{J_{\alpha}}{\rho}+2 V_{\alpha}\right]=0, \\
\partial_{t} J_{\alpha}+\partial_{\beta}\left\{P_{A \alpha \beta}+P_{B \alpha \beta}\right\}=0 .
\end{gathered}
$$

Equations (17) represent the conserved moments of the mixture. It is noted that the total momentum of the mixture is conserved by the present model. The nonconserved moments are obtained by adding the pressure Eq . (16) and by subtracting the momentum Eqs. (15):

$$
\begin{gathered}
\partial_{t} P_{\alpha \beta}+\partial_{\gamma} Q_{\alpha \beta \gamma}=\frac{1}{\tau_{1}}\left[P_{\alpha \beta}^{*}-P_{\alpha \beta}\right]+\frac{1}{\tau_{2}}\left[P_{\alpha \beta}^{\mathrm{eq}}-P_{\alpha \beta}^{*}\right], \\
\partial_{t}\left[\left(\rho_{A}-\rho_{B}\right) \frac{J_{\alpha}}{\rho}+2 V_{\alpha}\right]+\partial_{\beta}\left[P_{A \alpha \beta}-P_{B \alpha \beta}\right]=-\frac{2}{\tau_{2}} V_{\alpha},
\end{gathered}
$$

where $P_{\alpha \beta}=P_{A \alpha \beta}+P_{B \alpha \beta}, P_{\alpha \beta}^{*}=P_{A \alpha \beta}^{*}+P_{B \alpha \beta}^{*}$, and $P_{\alpha \beta}^{\mathrm{eq}}=P_{A \alpha \beta}^{\mathrm{eq}}$ $+P_{B \alpha \beta}^{\mathrm{eq}}$.

\section{CHAPMAN-ENSKOG EXPANSION}

The system of Eqs. (18) together with Eqs. (17) allows for the evaluation of $\tau_{1}$ and $\tau_{2}$ in the hydrodynamic limit. The standard procedure (Chapman-Enskog) is to expand all nonconserved variables, distribution functions, and time derivatives in Knudsen number series around the equilibrium:

$$
\begin{gathered}
f_{j i}=f_{j i}^{\mathrm{eq}}\left(\rho_{j}, \mathbf{U}\right)+\mathrm{Kn} f_{j i}^{(1)}+\mathrm{Kn}^{2} f_{j i}^{(2)}+\cdots, \\
\partial_{t}=\partial_{t}^{(0)}+\mathrm{Kn} \partial_{t}^{(1)}+\mathrm{Kn}^{2} \partial_{t}^{(2)}+\cdots, \\
V_{\alpha}=V_{\alpha}^{\mathrm{eq}}+\mathrm{Kn} V_{\alpha}^{(1)}+\mathrm{Kn}^{2} V_{\alpha}^{(2)}+\cdots, \\
P_{j \alpha \beta}=P_{j \alpha \beta}^{\mathrm{eq}}\left(\rho_{j}, \mathbf{J}\right)+\mathrm{Kn} P_{j \alpha \beta}^{(1)}+\mathrm{Kn}^{2} P_{j \alpha \beta}^{(2)}+\cdots, \\
P_{\alpha \beta}=P_{\alpha \beta}^{\mathrm{eq}}\left(\rho_{A}, \rho_{B}, \mathbf{J}\right)+\mathrm{Kn} P_{\alpha \beta}^{(1)}+\mathrm{Kn}^{2} P_{\alpha \beta}^{(2)}+\cdots .
\end{gathered}
$$

The diffusion flux $V_{\alpha}^{\mathrm{eq}}$ is zero, since at equilibrium the two components have the same velocity. The expansion is carried 
out with the further assumption that $\tau_{1}$ and $\tau_{2}$ are of $O(\mathrm{Kn})$. For the present model, the equilibrium pressure tensor and third-order moment, neglecting the cubic terms in $\mathbf{J}$, are

$$
\begin{gathered}
P_{\alpha \beta}^{\mathrm{eq}}\left(\rho_{A}, \rho_{B}, \mathbf{J}\right)=n k_{B} T_{0} \delta_{\alpha \beta}+\frac{J_{\alpha} J_{\beta}}{\rho} \\
Q_{\alpha \beta \gamma}^{\mathrm{eq}}\left(\rho_{A}, \rho_{B}, \mathbf{J}\right)=n k_{B} T_{0}\left(\frac{J_{\alpha}}{\rho} \delta_{\beta \gamma}+\frac{J_{\beta}}{\rho} \delta_{\alpha \gamma}+\frac{J_{\gamma}}{\rho} \delta_{\alpha \beta}\right)+O\left(\mathbf{J}^{3}\right),
\end{gathered}
$$

where $n=n_{A}+n_{B}$ is the total number of moles per unit volume, and $n_{j}=\rho_{j} / m_{j}$ is the number of moles of component $j$ per unit volume.

The pressure of component $j$ at the quasi-equilibrium $P_{j \alpha \beta}^{*}\left(\rho_{j}, \mathbf{J}_{j}\right)$ can be expressed as a function of the pressure at the equilibrium $P_{j \alpha \beta}^{\mathrm{eq}}\left(\rho_{j}, \mathbf{J}\right)$ through the expansion defined in Eqs. (19). Considering only the first-order terms of the expansion and using Eqs. (13):

$$
\begin{aligned}
& P_{A \alpha \beta}^{*}\left(\rho_{A}, \mathbf{J}_{A}\right)=P_{A \alpha \beta}^{\mathrm{eq}}\left(\rho_{A}, \mathbf{J}\right)+\operatorname{Kn}\left(\frac{1}{\rho} J_{\alpha} V_{\beta}^{(1)}+\frac{1}{\rho} J_{\beta} V_{\alpha}^{(1)}\right), \\
& P_{B \alpha \beta}^{*}\left(\rho_{B}, \mathbf{J}_{B}\right)=P_{B \alpha \beta}^{\mathrm{eq}}\left(\rho_{B}, \mathbf{J}\right)-\operatorname{Kn}\left(\frac{1}{\rho} J_{\alpha} V_{\beta}^{(1)}+\frac{1}{\rho} J_{\beta} V_{\alpha}^{(1)}\right) .
\end{aligned}
$$

The above equations also imply that $P_{A \alpha \beta}^{*}+P_{B \alpha \beta}^{*}=P_{A \alpha \beta}^{\mathrm{eq}}$ $+P_{B \alpha \beta}^{\mathrm{eq}}$.

The zeroth-order time derivatives for the conserved moments become

$$
\begin{gathered}
\partial_{t}^{(0)} \rho=-\partial_{\alpha} J_{\alpha}, \\
\partial_{t}^{(0)} J_{\alpha}=-\partial_{\beta}\left(n k_{B} T_{0} \delta_{\alpha \beta}+\frac{J_{\alpha} J_{\beta}}{\rho}\right), \\
\partial_{t}^{(0)}\left(\rho_{A}-\rho_{B}\right)=-\partial_{\alpha}\left[\left(\rho_{A}-\rho_{B}\right) \frac{J_{\alpha}}{\rho}\right] .
\end{gathered}
$$

Using Eqs. (19) to (23), the first-order expansions for the nonconserved moments are obtained from Eqs. (18)

$$
\begin{gathered}
\partial_{t}^{(0)} P_{\alpha \beta}^{\mathrm{eq}}+\partial_{\gamma} Q_{\alpha \beta \gamma}^{\mathrm{eq}}=-\frac{1}{\tau_{1}} P_{\alpha \beta}^{(1)}, \\
\partial_{t}^{(0)}\left[\left(\rho_{A}-\rho_{B}\right) \frac{J_{\alpha}}{\rho}\right]+\partial_{\beta}\left[\left(n_{A}-n_{B}\right) k_{B} T_{0} \delta_{\alpha \beta}\right] \\
+\partial_{\beta}\left[\left(\rho_{A}-\rho_{B}\right) \frac{J_{\alpha} J_{\beta}}{\rho^{2}}\right]=-\frac{2}{\tau_{2}} V_{\alpha}^{(1)} .
\end{gathered}
$$

\section{A. Viscosity}

The first of Eqs. (24) allows for the determination of the mixture viscosity at the hydrodynamic limit. Since $P_{\alpha \beta}^{\mathrm{eq}}$ is a function of $\rho_{A}, \rho_{B}$, and $\mathbf{J}$ :

$$
\partial_{t} P_{\alpha \beta}^{\mathrm{eq}}=\left(\partial_{t} \rho_{A}\right) \frac{\partial P_{\alpha \beta}^{\mathrm{eq}}}{\partial \rho_{A}}+\left(\partial_{t} \rho_{B}\right) \frac{\partial P_{\alpha \beta}^{\mathrm{eq}}}{\partial \rho_{B}}+\left(\partial_{t} J_{\gamma}\right) \frac{\partial P_{\alpha \beta}^{\mathrm{eq}}}{\partial J_{\gamma}} .
$$

Taking the derivatives with respect to $\rho_{A}, \rho_{B}, J_{\gamma}$, and $\theta$ (space) of Eq. (20):

$$
\begin{gathered}
\frac{\partial P_{\alpha \beta}^{\mathrm{eq}}}{\partial \rho_{A}}=\frac{1}{m_{A}} k_{B} T_{0} \delta_{\alpha \beta}-\frac{J_{\alpha} J_{\beta}}{\rho^{2}}, \\
\frac{\partial P_{\alpha \beta}^{\mathrm{eq}}}{\partial \rho_{B}}=\frac{1}{m_{B}} k_{B} T_{0} \delta_{\alpha \beta}-\frac{J_{\alpha} J_{\beta}}{\rho^{2}}, \\
\frac{\partial P_{\alpha \beta}^{\mathrm{eq}}}{\partial J_{\gamma}}=\frac{J_{\alpha}}{\rho} \delta_{\beta \gamma}+\frac{J_{\beta}}{\rho} \delta_{\alpha \gamma}, \\
\partial_{\theta} P_{\gamma \theta}^{\mathrm{eq}}=k_{B} T_{0} \delta_{\gamma \theta} \partial_{\theta} n+\frac{J_{\gamma}}{\rho} \partial_{\theta} J_{\theta}+\frac{J_{\theta}}{\rho} \partial_{\theta} J_{\gamma}-\frac{J_{\gamma} J_{\theta}}{\rho^{2}} \partial_{\theta} \rho .
\end{gathered}
$$

Substituting Eqs. (26) in Eq. (25), it is possible to express the time derivative of $P_{\alpha \beta}^{\mathrm{eq}}$, neglecting again the third-order terms in $\mathbf{J}$, as

$$
\begin{aligned}
\partial_{t}^{(0)} P_{\alpha \beta}^{\mathrm{eq}}= & -\partial_{\gamma}\left(\frac{n}{\rho} J_{\gamma}\right) k_{B} T_{0} \delta_{\alpha \beta}-k_{B} T_{0} \partial_{\gamma} n\left(\frac{J_{\alpha}}{\rho} \delta_{\beta \gamma}+\frac{J_{\beta}}{\rho} \delta_{\alpha \gamma}\right) \\
& +O\left(\mathbf{J}^{3}\right) .
\end{aligned}
$$

After some simplifications, the left-hand side of the first of Eqs. (24) becomes

$$
\partial_{t}^{(0)} P_{\alpha \beta}^{\mathrm{eq}}+\partial_{\gamma} Q_{\alpha \beta \gamma}^{\mathrm{eq}}=\left(\partial_{\alpha} \frac{J_{\beta}}{\rho}+\partial_{\beta} \frac{J_{\alpha}}{\rho}\right) n k_{B} T_{0},
$$

and equating with the corresponding right-hand side

$$
\left(\partial_{\alpha} \frac{J_{\beta}}{\rho}+\partial_{\beta} \frac{J_{\alpha}}{\rho}\right) n k_{B} T_{0}=\frac{1}{\tau_{1}} P_{\alpha \beta}^{(1)} .
$$

The pressure is obtained by adding the equilibrium and the nonequilibrium contributions, $P_{\alpha \beta}=P_{\alpha \beta}^{\mathrm{eq}}+\mathrm{Kn} P_{\alpha \beta}^{(1)}$. Substituting into the momentum equation [third of Eqs. (17)]

$$
\partial_{t} J_{\alpha}+\partial_{\alpha} P+\partial_{\beta}\left[\frac{J_{\alpha} J_{\beta}}{\rho}-\tau_{1} n k_{B} T_{0}\left(\partial_{\alpha} \frac{J_{\beta}}{\rho}+\partial_{\beta} \frac{J_{\alpha}}{\rho}\right)\right]=0,
$$

where $P=n k_{B} T_{0}$ is the static pressure of the system. By comparing it with the NS equations,

$$
\partial_{t} J_{\alpha}+\partial_{\alpha} P+\partial_{\beta}\left[\frac{J_{\alpha} J_{\beta}}{\rho}-\mu\left(\partial_{\alpha} \frac{J_{\beta}}{\rho}+\partial_{\beta} \frac{J_{\alpha}}{\rho}\right)\right]=0,
$$

the shear viscosity coefficient is finally recovered,

$$
\mu=n \tau_{1} k_{B} T_{0} .
$$

\section{B. Diffusion coefficient}

The second of Eqs. (24) allows for the extraction of the diffusion coefficient at the hydrodynamic limit. Using Eqs. (23) to eliminate the time derivatives in Eqs. (24), and after some rearrangements, 


$$
V_{\alpha}^{(1)}=\tau_{2}\left[\frac{\rho_{A}}{\rho} \partial_{\alpha} P-\partial_{\alpha} n_{A} k_{B} T_{0}\right]
$$

Noting that

$$
\partial_{\alpha} n_{A}=n\left(\partial_{\alpha} \frac{n_{A}}{n}+\frac{n_{A}}{n^{2}} \partial_{\alpha} n\right)
$$

the diffusion velocity can be written as

$$
\begin{aligned}
V_{\alpha}^{(1)} & =\tau_{2}\left[Y_{A} \partial_{\alpha} P-P\left(\partial_{\alpha} X_{A}+\frac{X_{A}}{P} \partial_{\alpha} P\right)\right] \\
& =\tau_{2} P\left[\left(Y_{A}-X_{A}\right) \frac{\partial_{\alpha} P}{P}-\partial_{\alpha} X_{A}\right],
\end{aligned}
$$

where $Y_{A}=\rho_{A} / \rho$ and $X_{A}=n_{A} / n$ are the mass and the mole fractions of component $A$, respectively. Rearranging Eq. (35), the molar fraction of species $A$ reads as

$$
\partial_{\alpha} X_{A}=-\frac{V_{\alpha}^{(1)}}{\tau_{2} P}+\left(Y_{A}-X_{A}\right) \frac{\partial_{\alpha} P}{P},
$$

and by comparing it with its macroscopic counterpart, the Stefan-Maxwell diffusion equation [16],

$$
\partial_{\alpha} X_{A}=\frac{X_{A} X_{B}}{D_{A B}}\left(\frac{J_{B \alpha}}{\rho_{B}}-\frac{J_{A \alpha}}{\rho_{A}}\right)+\left(Y_{A}-X_{A}\right) \frac{\partial_{\alpha} P}{P},
$$

the diffusion coefficient is recovered,

$$
D_{A B}=X_{A} X_{B} \frac{P}{m_{A B}} \tau_{2}
$$

It is now possible to analyze the restrictions on the Schmidt number,

$$
\mathrm{Sc}=\frac{\mu}{\rho D_{A B}}=\frac{\tau_{1}}{\tau_{2}} \frac{m_{A B}}{X_{A} X_{B}} \frac{1}{\rho},
$$

imposed by the present model. Considering that by construction $\tau_{1}<\tau_{2}$, the Schmidt number has to obey locally the following inequality:

$$
\mathrm{Sc}=\frac{\mu}{\rho D_{A B}} \leqslant \frac{Y_{A} Y_{B}}{X_{A} X_{B}} .
$$

As anticipated, this limitation stems from the fast-slow motion decomposition and the set of constraints used to maximize the entropy function in order to evaluate the quasiequilibrium distribution function $f^{*}\left(\rho_{j}, \mathbf{U}_{j}\right)$. Using as quasiconserved variable the stress tensor $P_{j \alpha \beta}$ of component $j$ to evaluate the quasi-equilibrium, a set of constraints for the minimization of the $H$-function can be obtained:

$$
\begin{gathered}
\rho_{j}=\sum_{i}^{N} f_{j i}, \\
J_{\alpha}=\sum_{j}^{A, B} \sum_{i}^{N} f_{j i} c_{j i \alpha},
\end{gathered}
$$

$$
P_{j \alpha \beta}=\sum_{i}^{N} f_{j i} c_{j i \alpha} c_{j i \beta}
$$

It is easy to show that by repeating the Chapman-Enskog expansion for this quasi-equilibrium $f^{* *}\left(\rho_{j}, \mathbf{U}, P_{j \alpha \beta}\right)$, the following is obtained:

$$
\mathrm{Sc} \geqslant \frac{Y_{A} Y_{B}}{X_{A} X_{B}}
$$

The solution to this minimization problem and the resulting quasi-equilibrium will be addressed in a future publication.

\section{NUMERICAL IMPLEMENTATION}

\section{A. Time integration}

In the lattice Boltzman scheme, Eqs. (1) are discretized in time by applying the implicit trapezoidal rule between time $t$ and $t+\delta t$ :

$$
\begin{aligned}
f_{j i}\left(\mathbf{x}+c_{j i} \delta t, t+\delta t\right)= & f_{j i}(\mathbf{x}, t)+\frac{\delta t}{2}\left[\Omega_{j i}(f(\mathbf{x}, t))+\Omega_{j i}(f(\mathbf{x}\right. \\
& \left.\left.\left.+c_{j i} \delta t, t+\delta t\right)\right)\right]+O\left(\delta t^{3}\right),
\end{aligned}
$$

with $\Omega_{j i}(f(\mathbf{x}, t))$ the collision integral at time $t$. This scheme is rendered explicit by introducing a local transformation through the auxiliary functions $g_{j i}$ such that

$$
g_{j i}(\mathbf{x}, t)=f_{j i}(\mathbf{x}, t)-\frac{\delta t}{2} \Omega_{j i}(f(\mathbf{x}, t)) .
$$

By substituting in Eq. (43) and using Eq. (2),

$$
\begin{aligned}
g_{j i}\left(\mathbf{x}+c_{j i} \delta t, t+\delta t\right)= & g_{j i}(\mathbf{x}, t)-\omega_{1}\left[g_{j i}(\mathbf{x}, t)-f^{*}\left(\rho_{j}, \mathbf{U}_{j}\right)\right] \\
& -\omega_{2}\left[f_{j i}\left(\rho_{j}, \mathbf{U}_{j}\right)-f_{j i}^{\mathrm{eq}}\left(\rho_{j}, \mathbf{U}\right)\right],
\end{aligned}
$$

with $\omega_{1}=2 \delta t /\left(2 \tau_{1}+\delta t\right)$ and $\omega_{2}=\omega_{1} \tau_{1} / \tau_{2}$. In this formulation $g_{j i}(t)$ is a function of both $f_{j i}^{*}(t, f)$ and $f_{j i}^{\mathrm{eq}}(t, f)$. Evaluating the moments of $g_{j i}(t)$ from Eq. (44) for the collision model under consideration yields

$$
\begin{gathered}
\rho_{j}(f)=\rho_{j}(g), \\
J_{j \alpha}(f)=\frac{\left(2 \tau_{2} / \delta t\right) J_{j \alpha}(g)+\rho_{j} / \rho J_{\alpha}(g)}{1+\left(2 \tau_{2} / \delta t\right)}, \\
J_{\alpha}(f)=J_{\alpha}(g) .
\end{gathered}
$$

The momentum $J_{j \alpha}$ of each individual species differs in the two distribution functions. This happens because the species momentum is not conserved by the collision term (a general feature of collision models for mixtures). The difference between $J_{j \alpha}(f)$ and $J_{j \alpha}(g)$ vanishes when the two masses are the same $\left(m_{A}=m_{B}\right)$, but becomes non-negligible when the mass ratio becomes large. This effect of discreteness, which leads to a redefinition of the nonconserved moments, was overlooked in previous works and led to lattice Boltzman methods that were first-order accurate in time $[11,17]$. The present discretization scheme allows for the transformation 
the initial implicit time integration problem in $f$ into an explicit equation in $g$. It is clarified that in order to evaluate $f^{\mathrm{eq}}(f)$ and $f^{*}(f)$, the moments must be computed using Eqs. (48). Moreover, it is emphasized that the time integration scheme is second-order accurate.

\section{B. Space discretization}

The time step is defined as: $d t=d x / c$, where $d x$ is the lattice spacing, and $c$ the lattice velocity. Since in a binary mixture the masses of the two components are generally dissimilar, the speed of sound of the two components will be different. In order to have the same time step for both components, two different lattice grids (one for each species) have to be used, and the corresponding lattice spacing ratio is related to the ratio of the sound speeds of the two components, i.e., to the inverse of the square root of the molecular weight ratio

$$
\frac{d x_{A}}{d x_{B}}=\frac{c_{A s}}{c_{B s}}=\sqrt{\frac{m_{B}}{m_{A}}} .
$$

This implies that the heavy component populations will reside on a finer grid or, equivalently, that the light component in one time step is diffusing faster than the heavier (Graham's effusion law). In a first implementation of the present model [12], a spacial discretization of Eqs. (45) on two different grids was used. Such a procedure has the advantage of keeping the streaming/advection step, resulting in a fast LB algorithm, but imposes restrictions on the molecular mass ratio, whose square root has to be a rational number since $d x_{j}$ are integers [see Eq. (47)]. Moreover, this implementation requires very fine grids when computing systems with a large mass ratio. In order to generalize the approach to any mass ratio and decrease the resolution requirements, an interpolation scheme using only one grid is implemented herein, as suggested in Refs. [11,18].

\section{MODEL APPLICATIONS}

The model was tested by simulating the time evolution of two classical diffusion problems: the interdiffusion of two components in a binary mixture subjected to an initial concentration gradient and the mixing of two flow streams having different inlet velocities and species concentrations.

\section{A. Binary diffusion}

In the binary diffusion case, the mixture consists of two different gases having a molecular mass ratio $m_{B} / m_{A}=500$ with an initial mole fraction concentration profile along the axial direction $x$ given by the step function:

$$
\begin{aligned}
& X_{A}=90 \%, \quad X_{B}=10 \% \quad \text { if } x<0, \\
& X_{A}=10 \%, \quad X_{B}=90 \% \quad \text { if } x \geqslant 0 .
\end{aligned}
$$

It is noted that the use of step functions is a severe test for the code compared to the use of smooth profiles. The boundary conditions are periodic on the horizontal boundaries, while on the vertical boundaries $(x= \pm L, L$ being the length

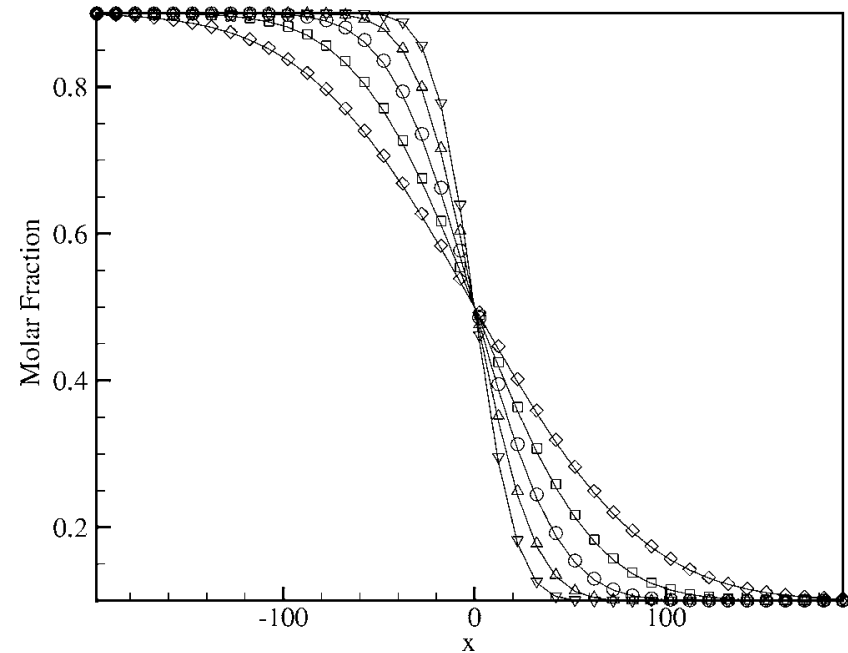

FIG. 1. Diffusion of a binary mixture, molar concentration of component $A$. Initial molar concentration $90 \% A-10 \% B$ for $x<0$ and $10 \% A-90 \% B$ for $x>0$, mass ratio $m_{B} / m_{A}=500$, and $D$ $=0.03$ in LB units. Symbols: LB predictions at time step 5000 (lower triangles); 10000 (upper triangles); 20000 (circles); 40000 (squares), and 80000 (diamonds). Continuous lines: corresponding analytical solution [Eq. (49)].

of the computational domain) the missing incoming populations are replaced by the quasi-equilibrium populations $f^{*}\left(\rho_{j}(t-1), 0\right)$, [19] with $\rho_{j}(t-1)$ the density of the component $j$ evaluated at the previous time step. The grid resolution is $500 \times 100$ lattice nodes along the diffusion direction $x$. The analytical time evolution for the mole fraction of component $j$ is $[20]$

$$
X_{j}=\left[\frac{1}{2}+\frac{\Delta X_{j}}{2} \operatorname{erf}\left(\frac{x}{\sqrt{4 D t}}\right)\right],
$$

where $\Delta X$ is the initial mole fraction difference, and $D$ is the diffusion coefficient. Figure 1 shows excellent agreement between the LB calculations and the analytical solution of Eq. (49). Despite the large mass ratio, the agreement persists for long-time integration (80000 time steps), indicating that no numerical diffusion is introduced by the interpolation scheme (the relative error is below 1\%). For the same mass ratio, it is possible to obtain a larger initial concentration difference $\left(X_{A}=99 \%, X_{B}=1 \%\right.$ and $\left.X_{A}=1 \%, X_{B}=99 \%\right)$ with a corresponding density ratio of ca. 60 , but in this case an initial smooth profile was necessary for numerical stability reasons.

\section{B. Laminar mixing layer}

The model is also tested on a dynamic diffusion process, the free-shear mixing of two initially separated binary fluid layers with velocities $U_{1}$ and $U_{2}$, and mass fractions $Y_{A 1}$ and $Y_{A 2}$, respectively (see Fig. 2). To the authors' knowledge there is only one work that tested a LB model with such a flow [21], in which the thickness growth of an unsteady mixing layer was compared with the experiments of Brown and Roshko [22]. Herein, this method is not followed since such a comparison involves the evaluation of the momentum thickness obtained by visual observation of the density pro- 


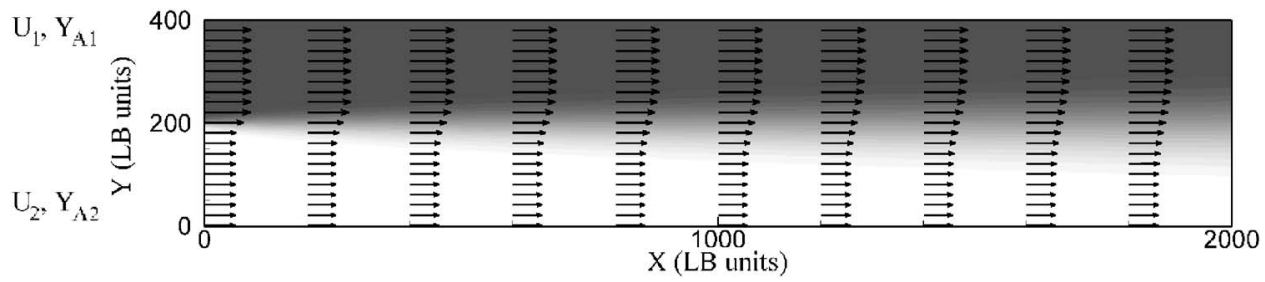

FIG. 2. Free-shear mixing. LB simulation: vector plot and concentration isocontours. Mass ratio $m_{B} / m_{A}=20$ and $\mathrm{Sc}_{1}=1.15$.

files leading to errors that can affect the assessment of the model accuracy. On the contrary, it is preferable to compare LB simulations with the predictions of a transient spectral element code that solves the continuum NS and species conservation equations and, in addition, with a similarity solution for the steady mixing layer. The comparison with a transient code is necessary to ensure that a steady solution is truly attainable. Linear stability analysis under the parallel flow assumption predicts that the mixing layer flow is unstable at any Reynolds number, $\operatorname{Re}$, where $\operatorname{Re}=U_{1} \delta / \nu_{1}$ and $\delta \approx\left(\nu_{1} x / U_{1}\right)^{1 / 2}$ is the boundary layer thickness (see for example Ref. [23]). Nevertheless, since this is a convectively unstable problem [24], i.e., disturbances are convected away downstream as they amplify in space, eventually leaving the basic flow undisturbed, the propagation of instabilities can be inhibited by simulating a flow at low Re number and by using a fine grid resolution in order to suppress the numerical noise that triggers instabilities. In such a way, a steady flow configuration can be obtained for the mixing layer.

The continuum conservation equations of total mass, momentum, and species are solved with a parallel code that uses spectral elements for the discretization of the spatial derivatives. The temporal discretization is based on a second-order mixed explicit/implicit operator splitting formulation [25]. The MPI-based parallel code runs with good scalability on a number of different platforms [26]. The computational domain was discretized with 1824 spectral elements and grid independence of the solution was ensured by using interpolating polynomial orders ranging from 4 to 10 in each spatial direction. Dirichlet boundary conditions are imposed on the inflow velocity, and flux boundary conditions for the species. At the outlet, zero Neumann boundary conditions are used for both the velocity and the species. Finally, on the lower and upper boundary, free-slip boundary conditions are enforced for the velocity and zero flux for the species.

\section{Similarity solution}

A similarity solution is derived herein, considering (in accordance to the LB formulation) constant dynamic viscosity $\mu$ and binary diffusion coefficient $D$. Invoking the boundary layer approximation $[16,20]$, the momentum and species conservation equations are

$$
\rho u \frac{\partial u}{\partial x}+\rho v \frac{\partial u}{\partial y}=\mu \frac{\partial^{2} u}{\partial y^{2}}, \quad \rho u \frac{\partial Y_{A}}{\partial x}+\rho v \frac{\partial Y_{A}}{\partial y}=\frac{\partial}{\partial y}\left(\rho D \frac{\partial Y_{A}}{\partial y}\right),
$$

with boundary conditions

$$
\begin{gathered}
y=\infty \quad u=U_{1}, Y_{A}=Y_{A 1}, \\
y=-\infty \quad u=U_{2}, Y_{A}=Y_{A 2} .
\end{gathered}
$$

The similarity solution is found by introducing the stream function $\psi$ such that

$$
\rho u=\rho_{1} \frac{\partial \psi}{\partial y}, \quad \rho v=-\rho_{1} \frac{\partial \psi}{\partial x} .
$$

A set of dependent and independent variables is defined

$$
\begin{gathered}
f=\frac{\psi}{\sqrt{2 \nu_{1} U_{1} x}}, \\
c_{A}=\frac{Y_{A}-Y_{A 1}}{Y_{A 2}-Y_{A 1}}, \\
\eta(x, y)=\sqrt{\frac{U_{1}}{2 \nu_{1} x}} \int \frac{\rho}{\rho_{1}} d y .
\end{gathered}
$$

Using Eqs. (52) and (53), Eqs. (50) can be rewritten as

$$
\begin{gathered}
\left(\frac{\rho}{\rho_{1}}\right) f^{\prime \prime \prime}+f f^{\prime \prime}+f^{\prime \prime} \frac{\partial}{\partial \eta}\left(\frac{\rho}{\rho_{1}}\right)=0, \\
\frac{\partial^{2} c_{A}}{\partial \eta^{2}}\left(\frac{\rho}{\rho_{1}}\right)^{2}+\operatorname{Sc}_{1} f \frac{\partial c_{A}}{\partial \eta}+\frac{\partial c_{A}}{\partial \eta} \frac{\partial}{\partial \eta}\left(\frac{\rho}{\rho_{1}}\right)^{2}=0,
\end{gathered}
$$

where $\mathrm{Sc}_{1}=\mu / \rho_{1} D \equiv \mu_{1} / \rho_{1} D_{1}$. The boundary conditions are

$$
\begin{gathered}
\eta=\infty, \quad f^{\prime}=1, c_{A}=0, \\
\eta=-\infty, \quad f^{\prime}=\frac{U_{2}}{U_{1}}, c_{A}=1, \\
\eta=0, \quad f=0 .
\end{gathered}
$$

One more equation is needed to evaluate $\rho / \rho_{1}$ as a function of the relative concentration $c_{A}$ in order to couple the momentum and the species equations. Following the previous formalism, the density can be expressed as

$$
\begin{aligned}
\rho=\rho_{A}+\rho_{B} & =m_{A} n_{A}+m_{B} n_{B} \\
& =n\left[m_{A} X_{A}+m_{B} X_{B}\right] \\
& =n\left[\left(m_{A}-m_{B}\right) X_{A}+m_{B}\right] .
\end{aligned}
$$

Similarly for the reference stream

$$
\rho_{1}=n_{1}\left[\left(m_{A}-m_{B}\right) X_{A 1}+m_{B}\right] .
$$

Since in the boundary layer approximation

$$
\frac{\partial P}{\partial y}=0
$$

and $P=n k_{B} T, n \equiv n_{1}$ must hold at any $y$, such that 

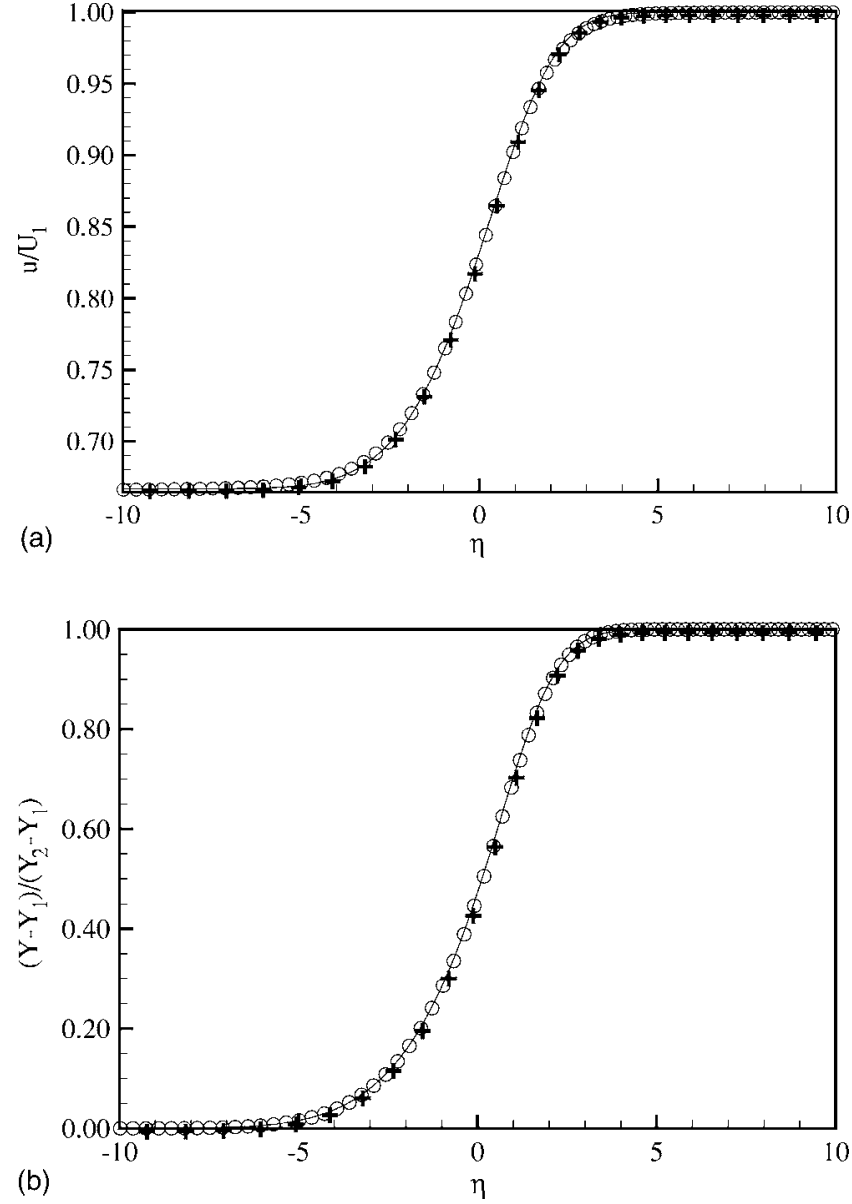

FIG. 3. Free-shear mixing. Comparison between the LB (circles), the spectral element code predictions (crosses), and the similarity solution (continuous lines) for: $m_{B} / m_{A}=20, \quad \mathrm{Sc}_{1}$ $=1.15, \mathrm{Sc}_{2}=0.69$. (a) Dimensionless velocity profiles. (b) Concentration profiles.

$$
\frac{\rho}{\rho_{1}}=\frac{\left(m_{A}-m_{B}\right) X_{A}+m_{B}}{\left(m_{A}-m_{B}\right) X_{A 1}+m_{B}} .
$$

Finally, the mole and the mass fractions are related according to

$$
X_{A}=\frac{Y_{A}}{m_{A}\left[\frac{Y_{A}}{m_{A}}+\left(\frac{1-Y_{A}}{m_{B}}\right)\right]} .
$$

The set of Eqs. (54), (59), and (60) subject to the boundary conditions of Eqs. (55) provides a similarity solution. The solution is obtained numerically with a finite-difference iterative scheme, using sufficiently long integration domains $(\eta= \pm 24)$.

\section{Comparison}

The LB computational domain is a grid of $400 \times 2000$ nodes (see Fig. 2). Free-slip boundary conditions are applied at the horizontal boundaries. The similarity boundary conditions, which were imposed under the assumption of infinite domain, cannot be used both in the LB and spectral element code, since they are creating a transverse flow that renders computations unstable. At the outlet the missing incoming populations are replaced by the extrapolated quasiequilibrium populations from the previous node, while at the inlet the missing incoming populations are replaced by the equilibrium populations $f^{\text {eq }}$ corresponding to the imposed inlet conditions for concentration and velocity profiles:

$$
\begin{aligned}
& X_{A}=90 \%, \quad X_{B}=10 \%, \quad U=U_{1} \quad \text { if } y>\frac{L_{y}}{2}, \\
& X_{A}=80 \%, \quad X_{B}=20 \%, \quad U=U_{2} \quad \text { if } y \geqslant \frac{L_{y}}{2}
\end{aligned}
$$

where $U_{2}=\frac{2}{3} U_{1}$ and $L_{y}$ is the total number of nodes in the transverse direction. The simulations are performed for a mass ratio of $m_{B} / m_{A}=20$. The concentrations are chosen in order to satisfy the restrictions of Eq. (40) and, at the same time, to have a large viscosity $\nu$ (low Re number) by keeping the concentration of the heavy component small. The resulting Schmidt number was in the range $1.15\left(=\mathrm{Sc}_{1}\right)>\mathrm{Sc}$ $>0.69\left(=\mathrm{Sc}_{2}\right)$. The results obtained with the LB model, the spectral element solution of the continuum equations, and the similarity solution are compared in Fig. 3. It is emphasized that the LB and spectral element solution points in Fig. 3 pertain to various axial positions. The three results are in excellent agreement regarding both the axial velocity and the concentration profiles. The very good agreement is also maintained for the same mass ratio, when the Sc range is decreased to $0.69\left(=\mathrm{Sc}_{1}\right)>\mathrm{Sc}>0.41\left(=\mathrm{Sc}_{2}\right)$, and when changing the mass ratio to $m_{B} / m_{A}=16$ with $1.33\left(=\mathrm{Sc}_{1}\right)>\mathrm{Sc}$ $>0.833\left(=\mathrm{Sc}_{2}\right)$ (for clarity those results are not shown in Fig. 3).

A further assessment of the model accuracy and its implementation is performed by comparing the transverse velocity $v$. Since the magnitude of $v$ is considerably smaller than the reference velocity $U_{1}$ (by a factor of $10^{-3}$ ), its value is very sensitive to the applied boundary conditions. The simulation

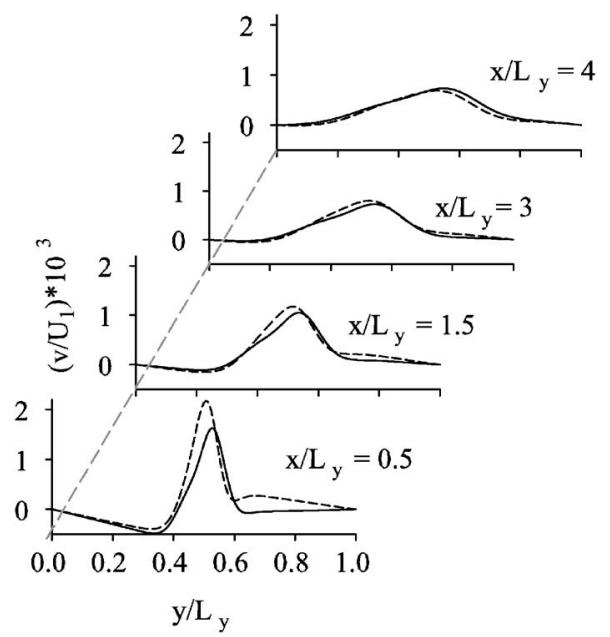

FIG. 4. Free-shear mixing. Predictions of the transverse velocity at different sections for $m_{B} / m_{A}=20, \mathrm{Sc}_{1}=1.15$, and $\mathrm{Sc}_{2}=0.69$. Continuous line: LB. Dashed line: spectral element code predictions. 


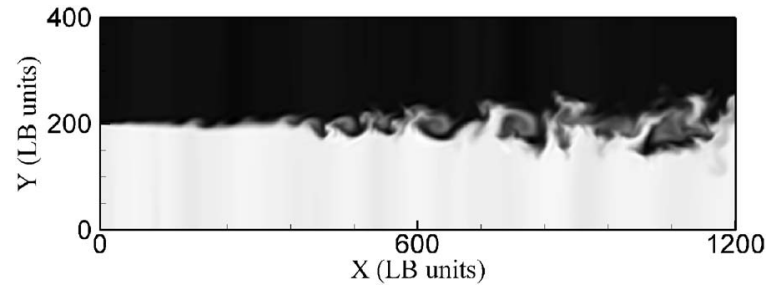

FIG. 5. Free-shear mixing. LB simulation. Instantaneous concentration profile for the nonsteady case, $m_{B} / m_{A}=16, \mathrm{Sc}_{1}=1.33$, and $\mathrm{Sc}_{2}=0.833$.

has to be performed using smooth profiles (hyperbolic tangent) for the axial velocity and concentration at the inlet in order to suppress any subsequent instability that can affect the transverse velocity. Figure 4 shows comparisons of the transverse velocity at different axial positions. The agreement is quite good far from the inlet (the error in the prediction of the maximum is around $6 \%$ for $x / L_{y}=4$ ). Close to the inlet the differences in the applied boundary conditions result in larger discrepancies (ca. $25 \%$ at $x / L_{y}=0.5$ ).

The developed model applies not only to steady, but also to unsteady flows. As an example of the capacity of the LB code to simulate nonsteady flows at higher Re numbers, the previous calculation with $m_{B} / m_{A}=16$ and $1.33\left(=\mathrm{Sc}_{1}\right)>\mathrm{Sc}$ $>0.833\left(=\mathrm{Sc}_{2}\right)$ is repeated by decreasing the dynamic viscosity $\mu$ from $5 \times 10^{-3}$ to $5 \times 10^{-4}$. The concentration profiles are presented in Fig. 5. Details on the jet instabilities and comparison with the spectral element code, however, are outside the scope of the present work.

\section{CONCLUSIONS}

The LB model based on the quasi-equilibrium formulation presented in this paper allows for the simulation of binary mixtures with any molecular mass ratio and a wide range of Schmidt numbers. It has been shown that both the Navier-Stokes and the Stefan-Maxwell diffusion equations are recovered in the hydrodynamic limit. The corresponding viscosity and diffusion coefficients are related to the relaxation times towards the quasi-equilibrium, $\tau_{1}$, and the equi- librium, $\tau_{2}$, by: $\mu=n \tau_{1} k_{B} T_{0}$ and $D_{A B}=X_{A} X_{B} P / m_{A B} \tau_{2}$. Despite the simplicity of the presented model (its implementation on top of the standard LBGK code is straightforward), it is free of the drawbacks of previous models, and is thermodynamically consistent. Applications to binary diffusion and mixing layers were presented. The LB predictions for a laminar flow were in good agreement with the solution of the continuum conservation equations obtained by a spectral element code and with a similarity solution.

\section{ACKNOWLEDGMENT}

I.V.K. was supported by the BFE-Project No. 100862. Helpful discussions with Professor A. Tomboulides are gratefully acknowledged.

\section{APPENDIX: $H$-THEOREM}

The lattice Boltzman equation for the binary mixture reads as

$$
\partial_{t} f_{i j}+c_{i j \alpha} \partial_{\alpha} f_{i j}=-\frac{1}{\tau_{1}}\left(f_{i j}-f_{i j}^{*}\right)-\frac{1}{\tau_{2}}\left(f_{i j}^{*}-f_{i j}^{\mathrm{eq}}\right) .
$$

By adding and subtracting to the right-hand side the term $\left(1 / \tau_{2}\right) f_{i j}, j=A, B$ :

$$
\partial_{t} f_{i j}+c_{i j \alpha} \partial_{\alpha} f_{i j}=-\frac{1}{\tau_{2}}\left(f_{i j}-f_{i}^{\mathrm{eq}}\right)-\left(\frac{1}{\tau_{1}}-\frac{1}{\tau_{2}}\right)\left(f_{i j}-f_{i j}^{*}\right)
$$

For the present model, the $H$ function is defined as

$$
H=\sum_{j}^{A, B} \sum_{i}^{N} f_{i j} \ln \frac{f_{i j}}{W_{i}}
$$

where $W_{i}$ are the weights defined in Eq. (6). The $H$ function transport equation is obtained by multiplying Eqs. (A2) by $\ln f_{i j} / W_{i}$, and summing up over $i=1, \ldots, N$, for both components $A$ and $B$,

$$
\partial_{t} H+\partial_{\alpha} J_{\mathrm{H} \alpha}=-\frac{1}{\tau_{2}} \sum_{i}^{N} \ln \frac{f_{i A}}{W_{i}}\left(f_{i A}-f_{i A}^{\mathrm{eq}}\right)-\left(\frac{1}{\tau_{1}}-\frac{1}{\tau_{2}}\right) \sum_{i}^{N} \ln \frac{f_{i A}}{W_{i}}\left(f_{i A}-f_{i A}^{*}\right)-\frac{1}{\tau_{2}} \sum_{i}^{N} \ln \frac{f_{i B}}{W_{i}}\left(f_{i B}-f_{i B}^{\mathrm{eq}}\right)-\left(\frac{1}{\tau_{1}}-\frac{1}{\tau_{2}}\right) \sum_{i}^{N} \ln \frac{f_{i B}}{W_{i}}\left(f_{i B}-f_{i B}^{*}\right),
$$

where

$$
J_{\mathrm{H} \alpha}=\sum_{j}^{A, B} \sum_{i}^{N} c_{i j \alpha} f_{i j} \ln \frac{f_{i j}}{W_{i}}
$$

is the $H$-flux. Using the conservation laws,

$$
\sum_{i}^{N}\left(f_{i j}-f_{i j}^{\mathrm{eq}}\right)=0, \quad \sum_{i}^{N}\left(f_{i j}-f_{i j}^{*}\right)=0
$$




$$
\sum_{j}^{A, B} \sum_{i}^{N}\left(f_{i j}-f_{i j}^{\mathrm{eq}}\right) c_{i j \alpha}=0, \quad \sum_{i}^{N}\left(f_{i j}-f_{i j}^{*}\right) c_{i j \alpha}=0,
$$

and considering that $\ln \left(f_{i j}^{\mathrm{eq}}\right)=A+B c_{i j}$ and $\ln \left(f_{i j}^{*}\right)=C+D c_{i j}$, where $A, B, C$, and $D$ are Lagrange multipliers,

$$
\begin{aligned}
& \sum_{i}^{N}\left(f_{i j}-f_{i j}^{\mathrm{eq}}\right) \ln \left(f_{i j}^{\mathrm{eq}}\right)=0, \\
& \sum_{i}^{N}\left(f_{i j}-f_{i j}^{*}\right) \ln \left(f_{i j}^{*}\right)=0 .
\end{aligned}
$$

By adding the last two terms to Eq. (A4) for each component

$$
\partial_{t} H+\partial_{\alpha} J_{\mathrm{H}}=-\frac{1}{\tau_{2}} \sum_{i}^{N} \ln \frac{f_{i A}}{f_{i A}^{\mathrm{eq}}}\left(f_{i A}-f_{i A}^{\mathrm{eq}}\right)-\left(\frac{1}{\tau_{1}}-\frac{1}{\tau_{2}}\right) \sum_{i}^{N} \ln \frac{f_{i A}}{f_{i A}^{*}}\left(f_{i A}-f_{i A}^{*}\right)-\frac{1}{\tau_{2}} \sum_{i}^{N} \ln \frac{f_{i B}}{f_{i B}^{\mathrm{eq}}}\left(f_{i B}-f_{i B}^{\mathrm{eq}}\right)-\left(\frac{1}{\tau_{1}}-\frac{1}{\tau_{2}}\right) \sum_{i}^{N} \ln \frac{f_{i B}}{f_{i B}^{*}}\left(f_{i B}-f_{i B}^{*}\right) .
$$

Considering that the quantities $\ln f_{i j} / f_{i j}^{\mathrm{eq}}\left(f_{i j}-f_{i j}^{\mathrm{eq}}\right)$ and $\ln f_{i j} / f_{i j}^{*}\left(f_{i j}-f_{i j}^{*}\right)$ are always non-negative-definite, a sufficient condition to have a non-negative-definite entropy production $E=-k_{B} H$ is $\tau_{2} \geqslant \tau_{1}>0$, and the $H$ theorem is obtained.

[1] F. J. Higuera, S. Succi, and R. Benzi, Europhys. Lett. 9, 345 (1989).

[2] Y. H. Qian, D. d'Humieres, and P. Lallemand, Europhys. Lett. 17, 479 (1992).

[3] I. V. Karlin, A. N. Gorban, S. Succi, and V. Boffi, Phys. Rev. Lett. 81, 6 (1998).

[4] S. Ansumali and I. V. Karlin, Phys. Rev. E 65, 056312 (2002).

[5] S. Ansumali, I. V. Karlin, and H. C. Öttinger, Europhys. Lett. 63, 798 (2003).

[6] S. Ansumali and I. V. Karlin, Phys. Rev. Lett. 95, 260605 (2005).

[7] I. V. Karlin, A. Ferrante, and H. C. Ottinger, Europhys. Lett. 47, 182 (1999).

[8] A. N. Gorban and I. V. Karlin, Invariant Manifolds for Physical and Chemical Kinetics (Springer, Berlin, 2005).

[9] P. Asinari, Phys. Fluids 17, 067102 (2005).

[10] P. Andries, K. Aoki, and B. Perthame, J. Stat. Phys. 106, 993 (2002).

[11] M. E. McCracken and J. Abraham, Phys. Rev. E 71, 046704 (2005).

[12] S. Arcidiacono, S. Ansumali, I. V. Karlin, J. Mantzaras, and K.

B. Boulouchos, Math. Comput. Simul. 72, 79 (2006).

[13] S. Ansumali and I. V. Karlin, Phys. Rev. E 65, 056312 (2002).

[14] A. N. Gorban and I. V. Karlin, Physica A 206, 401 (1994).
[15] C. D. Levermore, J. Stat. Phys. 83, 1021 (1996).

[16] F. Williams, Combustion Theory (Benjamin/Cumming, California, 1986).

[17] L. S. Luo and S. S. Girimaji, Phys. Rev. E 67, 036302 (2003).

[18] H. R. Wu, Y. L. He, G. H. Tang, and W. Q. Tao, Progr. Comput. Fluid Dyn. 5, 97 (2005).

[19] S. S. Chikatamarla, S. Ansumali, and I. V. Karlin, Europhys. Lett. 74, 215 (2006).

[20] F. P. Incropera and D. P. DeWitt, Fundamentals of Heat and Mass Transfer (Wiley, New York, 2002).

[21] M. E. McCracken and J. Abraham, Int. J. Mod. Phys. E 16, 533547 (2005).

[22] G. L. Brown and A. Roshko, J. Fluid Mech. 64, 775 (1974).

[23] A. Öztekin, L. J. Cumbo, and A. Liakopoulos, Theor. Comput. Fluid Dyn. 13, 77 (1999).

[24] P. Huerre and P. A. Monkewitz, J. Fluid Mech. 159, 151 (1985).

[25] G. E. Karniadakis, M. Israeli, and S. A. Orszag, J. Comput. Phys. 97, 414 (1991).

[26] P. F. Fischer and H. M. Tufo, High-performance Spectral Element Algorithms and Implementations in Parallel Computational Fluid Dynamics: Towards Teraflops, Optimization and Novel Formulations, A. Ecer, N. Satofuka, P. Fox, and J. Periaux (North-Holland, Amsterdam, 2000), pp. 17-26. 